\title{
Improvement in Medication Adherence after Pharmacist Intervention Is Associated with Favorable Clinical Outcomes in Patients with Ulcerative Colitis
}

\author{
Jae Song Kim,2, Min Jung Geum¹, Eun Sun Son ${ }^{1,2}$, Yun Mi Yư ${ }^{3}$, Jae Hee Cheon ${ }^{4}$, and Kyeng Hee Kwon² \\ ${ }^{1}$ Department of Pharmacy, Severance Hospital, Yonsei University Health System, Seoul, ${ }^{2}$ College of Pharmacy, Dongguk University \\ Biomedi Campus, Goyang, ${ }^{3}$ Department of Pharmacy and Yonsei Institute of Pharmaceutical Sciences, College of Pharmacy, Yonsei \\ University, Incheon, and ${ }^{4}$ Department of Internal Medicine and Institute of Gastroenterology, Yonsei University College of Medicine, \\ Seoul, Korea
}

See editorial on page 665.

\section{Article Info}

Received August 14, 2021

Revised October 23, 2021

Accepted November 3, 2021

Published online February 11, 2022

\section{Corresponding Author}

\section{Jae Hee Cheon}

ORCID https://orcid.org/0000-0002-2282-8904

E-mail GENIUSHEE@yuhs.ac

Kyeng Hee Kwon

ORCID https://orcid.org/0000-0003-2421-4030

E-mail khkwon@dongguk.edu
Background/Aims: Although pharmacist intervention for patients with chronic diseases has been shown to improve medication adherence, few studies have evaluated its effects on the objective clinical outcomes. We investigated the impact of pharmacist intervention on medication adherence and clinical outcomes in patients with ulcerative colitis (UC).

Methods: Patients with UC and low medication adherence were divided into two groups, based on pharmacist intervention. Their medication possession ratio and nonadherence rate for 6 months before and after the baseline were investigated. The partial Mayo score, flare-up incidence, and factors influencing flare-up events for 1 year after the baseline were analyzed.

Results: Of 99 patients, 33 and 66 were included in the intervention and control groups, respectively. The nonadherence rate significantly declined in the intervention group 6 months after the baseline $(60.6 \%$ before vs $30.3 \%$ after; $p=0.013)$. The groups showed a significant difference regarding time-related partial Mayo scores $(p=0.002)$. Intervention was significantly negatively correlated with time and the partial Mayo score $\left(r^{2}=0.035, p=0.013\right)$. A significant difference was observed in the flare-up incidence $33.3 \%$ in the intervention group vs $54.6 \%$ in the control group; $p=0.046$ ). Multivariate logistic regression indicated that pharmacist intervention (adjusted odds ratio, $0.370 ; 95 \%$ confidence interval, 0.145 to $0.945 ; p=0.038$ ) independently reduced the flareup risk.

Conclusions: Pharmacist intervention significantly decreased the nonadherence rate, improved the partial Mayo score, and reduced the flare-up incidence compared with the control group in a cohort of UC patients identified to have low medication adherence. (Gut Liver 2022;16:736-745)

Key Words: Counseling; Medication adherence; Pharmacists; Recurrence; Ulcerative colitis

\section{INTRODUCTION}

Ulcerative colitis (UC) is an inflammatory bowel disease (IBD) characterized by continuous mucosal inflammation that commences in the rectum and extends towards the colon. ${ }^{1,2}$ In 2020, South Korea had the second-highest incidence and prevalence rates of IBD in East Asia. ${ }^{3}$ Further, the cost of managing patients with IBD has increased over time. ${ }^{4,5} \mathrm{UC}$ is a lifelong disease characterized by recurrent remissions and unpredictable flare-ups, with a consider- able number of patients unable to maintain a continuous state of remission. ${ }^{6,7}$ The goal of UC treatment is to induce and maintain corticosteroid-free remission. ${ }^{8,9}$ However, $>80 \%$ of patients with UC experience a minimum of one flare-up during the decade following the diagnosis. ${ }^{10}$ The IBSEN study suggested that the maintenance of remission after prior remission induction is necessary to prevent further flare-ups. ${ }^{10}$

Pharmacotherapy is the basis of the treatment for patients with $\mathrm{UC},{ }^{11}$ and medication adherence is one of the 
key factors associated with remission maintenance. ${ }^{12}$ However, according to a systematic literature review, $30 \%$ to $45 \%$ of patients with UC were reported to be among those with low medication adherence..$^{13}$ Low medication adherence in patients with UC can lead to increased disease activity, loss of treatment response, and increased treatment cost. ${ }^{14,15}$ Several studies have demonstrated that pharmacist interventions in patients with chronic diseases, including IBD, can improve their medication adherence. ${ }^{16-18}$ Tiao et al..$^{19}$ reported that medication adherence of patients with UC was improved through personalized counseling by pharmacists.

Most previous studies regarding pharmacist intervention in patients with UC have only investigated the improvement in medication adherence and suggested performance tasks and roles for pharmacists. ${ }^{19-21}$ Few studies have evaluated the effects of pharmacist intervention on objective clinical outcomes. Therefore, we analyzed the effects of pharmacist interventions on both medication adherence and clinical outcomes in patients with UC with low adherence, compared with a control group of similar patients without intervention.

\section{MATERIALS AND METHODS}

This was a retrospective longitudinal study using data extracted from the electronic medical records (EMRs) at a single tertiary hospital in South Korea. This study conformed with the Ethical Guidelines of the Helsinki Declaration in 1975. The study protocol was approved by the Institutional Review Board of the Yonsei University Health System (IRB number: 4-2021-0251). The informed consent was waived.

\section{Patients and study design}

The study population comprised only those who were identified as "non-adherers" in the EMR. The subjects were divided into two groups. During the study enrollment period (from October 2017 to April 2019), nonadherent patients were allocated to an intervention group if they were referred to a pharmacist for medication counseling. Patients were allocated to the control group if they were recorded as non-adherers in the EMR, but had not been referred to a pharmacist. Non-adherer in our medical institution is recorded when more than $30 \%$ of the medication prescribed on the previous clinic visit day remains unused on the following visit day, which is based on staff opinion and consensus.

The inclusion criteria were as follows: (1) aged $\geq 19$ years; (2) diagnosed with UC at least 6 months before the study enrollment (International Classification of Diseases, Tenth Revision code: K51); (3) undergoing outpatient treatment with a specific gastroenterologist; (4) a clear record of nonadherence on the EMR; (5) having prescriptions for topical medications related to UC treatment or patients in a non-remission state (baseline partial Mayo score [PMS] $\geq 3)^{22}$ that warranted intervention by a pharmacist; or (6) having follow-up records spanning a minimum of 8 months after enrollment in the study. Patients who met more than one of the following exclusion criteria were excluded from the study: (1) diagnosed with simultaneous UC and Crohn's disease; (2) having insufficient baseline scores on PMS-related items; (3) not having prescriptions for topical medication and with baseline PMS $\leq 2$; (4) having fewer than two outpatient clinic visits during the follow-up period after the baseline; (5) participation in other clinical studies; (6) had received counseling by a pharmacist before the study period; (7) pregnancy; (8) solid organ transplant recipients; (9) not receiving medications; or (10) with malignant tumors.

\section{The procedure for pharmacist intervention}

The IBD multidisciplinary team at our hospital comprises doctors, pharmacists, nurses, nutritionists, and social workers. The investigators developed the following counseling protocol for outpatients with UC who need to improve medication adherence: Patients who have been identified as having low medication adherence by physicians were referred to a pharmacist for medication consultation. After the next request for consultation, the pharmacist reviewed patient information and prescriptions, then prepared consultation materials, such as medication information brochures and medication schedules. The pharmacist subsequently conducted educational interventions, such as personalized counseling regarding medication dosage, drug mechanisms of action, effects, and possible side effects, as well as coping/prevention methods, associated discomfort, difficulties, and concerns related to medication use. Each consultation with the pharmacist took approximately 30 minutes. All consultations were free. If the patient agreed to a 1-week follow-up telephone consultation, the pharmacist proceeded with a second consultation at the scheduled time with the patient's phone number (Fig. 1).

\section{Clinical outcomes}

1) Comparison of medication adherence: medication possession ratio and nonadherence rate

The medication possession ratio (MPR) was defined as a value obtained by dividing the total number of prescribed days of the administration of medications during 


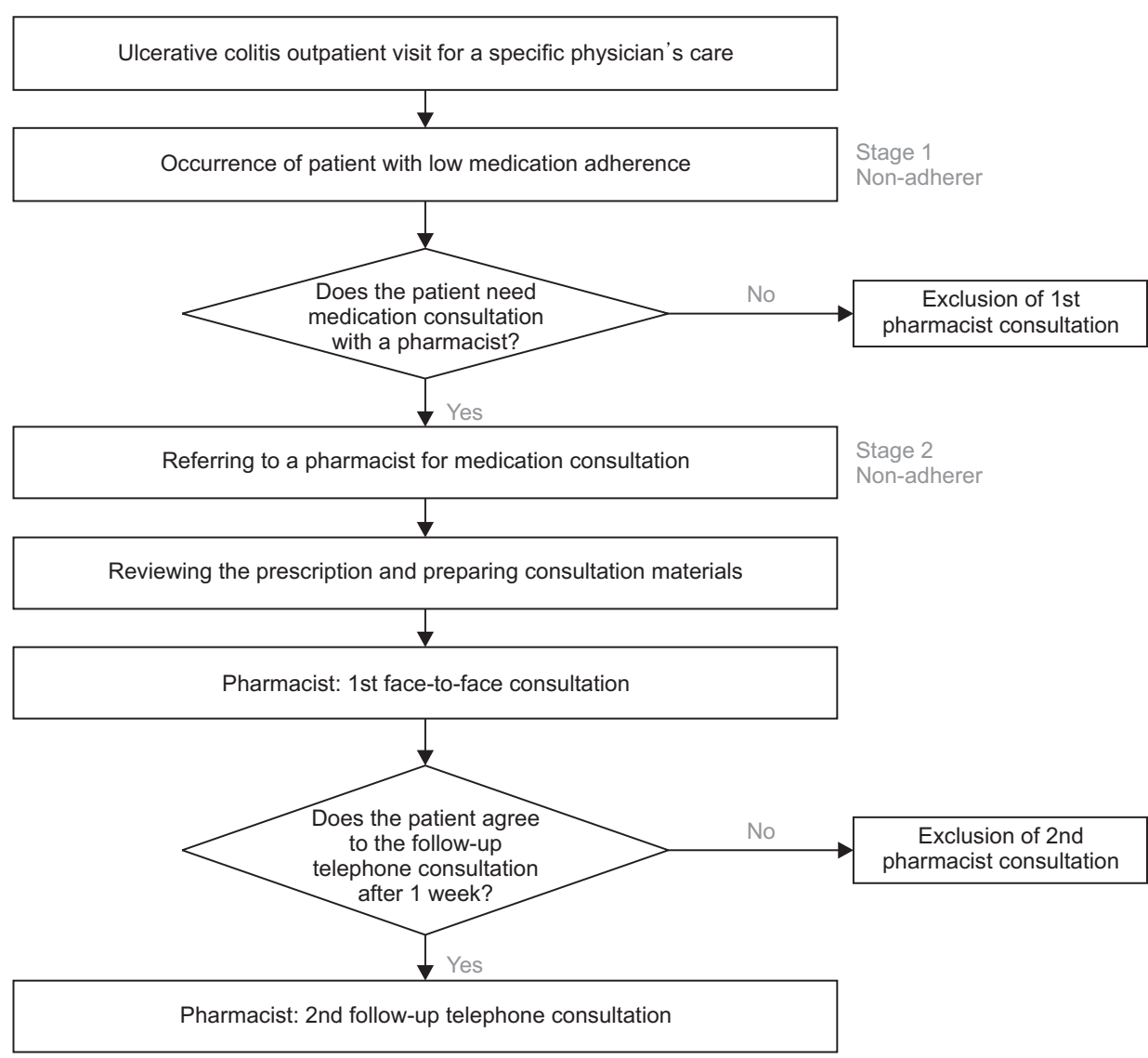

Fig. 1. The procedure of pharmacist intervention. the follow-up period by the number of days of follow-up. ${ }^{15}$ Nonadherence was defined as MPR $<80 \% .{ }^{15}$ When multiple treatments were prescribed for the management of UC, the MPR was calculated as the maximum number of days of prescription among the concomitant medications. The baseline MPR was calculated during an observation period of 6 months. The MPR values between the two groups were compared before and after the baseline following another 6-month observation period. The two groups were also compared regarding the MPR for a 1-year postbaseline observation period and regarding the change in the MPR between 6-month pre-baseline and 1-year postbaseline.

\section{2) Comparison of disease activity: time-related improvement in the PMS}

We collected data regarding the frequency of stools, rectal bleeding, and the physician's global assessment to compute the PMS over 1 year after enrollment in this study. ${ }^{23}$ This study statistically analyzed the PMS over time at 1-month intervals to evaluate the degree of improvement. Subsequently, linear regression analysis was performed on each group to estimate the regression equation between time and the PMS.

\section{3) Comparison of the flare-up incidence rates}

A flare-up was defined as having one or more occurrences of the following items: dose escalation of medications used for UC treatment, step-up therapy, the addition of corticosteroids, ${ }^{10,24}$ and the occurrence of severe disease activity (PMS $\geq 5$ ) at least once during the follow-up period. ${ }^{25}$ The two groups were compared regarding the rate of flare-up.

\section{4) Comparison of other items}

The rates of hospitalization, surgery, and emergency room visits, the defined daily dose of the corticosteroids administered during the 1-year period, and factors related to the flare-up incidence were analyzed. The number of corticosteroids used by the participants was expressed using the defined daily dose, which is the average daily maintenance dose of the medication, as developed by the World Health Organization (Supplementary Table 1) ${ }^{26}$

\section{Statistical analyses}

The baseline patient characteristics of the control and intervention groups were summarized via descriptive statistics and depending on the variables, were compared using the t-test or the Mann-Whitney $\mathrm{U}$ test and the chisquare test or the Fisher exact test. The significance level 
was set at $\mathrm{p}<0.05$. Data analysis was performed using SPSS version 25 (IBM Corp., Armonk, NY, USA) and R version 4.0.0 (R Foundation for Statistical Computing, Vienna, Austria).

Propensity score matching (PSM) was performed using the optimal PSM method in the intervention and control groups at a ratio of 1:2. The covariates included sex, age at baseline, age at diagnosis, time elapsed after diagnosis, baseline PMS, remission status, oral 5-aminosalicylate use, biologics use, and disease extent. A linear mixed model was used to analyze the group-by-time interaction regarding the PMS at 1-month intervals. When the PMS was evaluated twice or more within 1 month, the highest score was used.

The flare-up incidence rates were compared between the two groups. Univariate and multivariate analyses were performed to analyze the factors related to flare-ups. A multivariate logistic regression analysis was performed in accordance with the forward stepwise selection (likelihood ratio) procedure for any factors that yielded results of $\mathrm{p}<0.2$ in the univariate analysis.

The Kaplan-Meier analysis was performed to identify the effect of pharmacist intervention on the time of occurrence of flare-ups during the follow-up period.

\section{RESULTS}

\section{Basic participant characteristics}

The number of patients who met the inclusion criteria from October 2017 to April 2019 was 54 in the interven- tion group and 279 in the control group-333 patients in total. Among these, 21 in the intervention group and 149 in the control group were excluded. The study group finally comprised 33 in the intervention group and 130 in the control group. PSM was subsequently performed at a ratio of 1:2, with 66 of the 130 control participants selected. Thus, the number of participants finally included in this study was 99 (Fig. 2).

The median age of the participants was 33.0 years (interquartile range [IQR], 26.1 to 41.5 ), with $60.6 \%$ being male; their median age at the time of diagnosis was 29.1 years (IQR, 22.7 to 36.6). The median time interval after diagnosis was 3.6 years (IQR, 1.5 to 4.9 ).

No significant differences were found between the intervention $(n=33)$ and control groups $(n=66)$ regarding baseline demographic distributions or regarding the following: median PMS ( $\mathrm{p}=0.194)$, number of patients with PMS $\leq 2(\mathrm{p}=0.444)$, number of patients using external medications $(\mathrm{p}=1.000)$, and number of patients undergoing corticosteroid therapy within 1 year before their enrollment in this study $(\mathrm{p}=0.269)$. There were no statistically significant differences between the two groups regarding the disease extent and rate of biologics use (Table 1).

\section{Comparison of the MPRs and nonadherence rates}

There was no significant difference between the two groups regarding their median MPRs (control group, $90.8 \%$ [IQR, $77.1 \%$ to $100 \%$ ] vs intervention group, $86.3 \%$ [IQR, $75.2 \%$ to $96.2 \%$ ]; $\mathrm{p}=0.254$ ) and nonadherence rates (30.3\% vs $39.4 \%$; $\mathrm{p}=0.366$ ) for the 1 -year observation period after the baseline (Table 2).

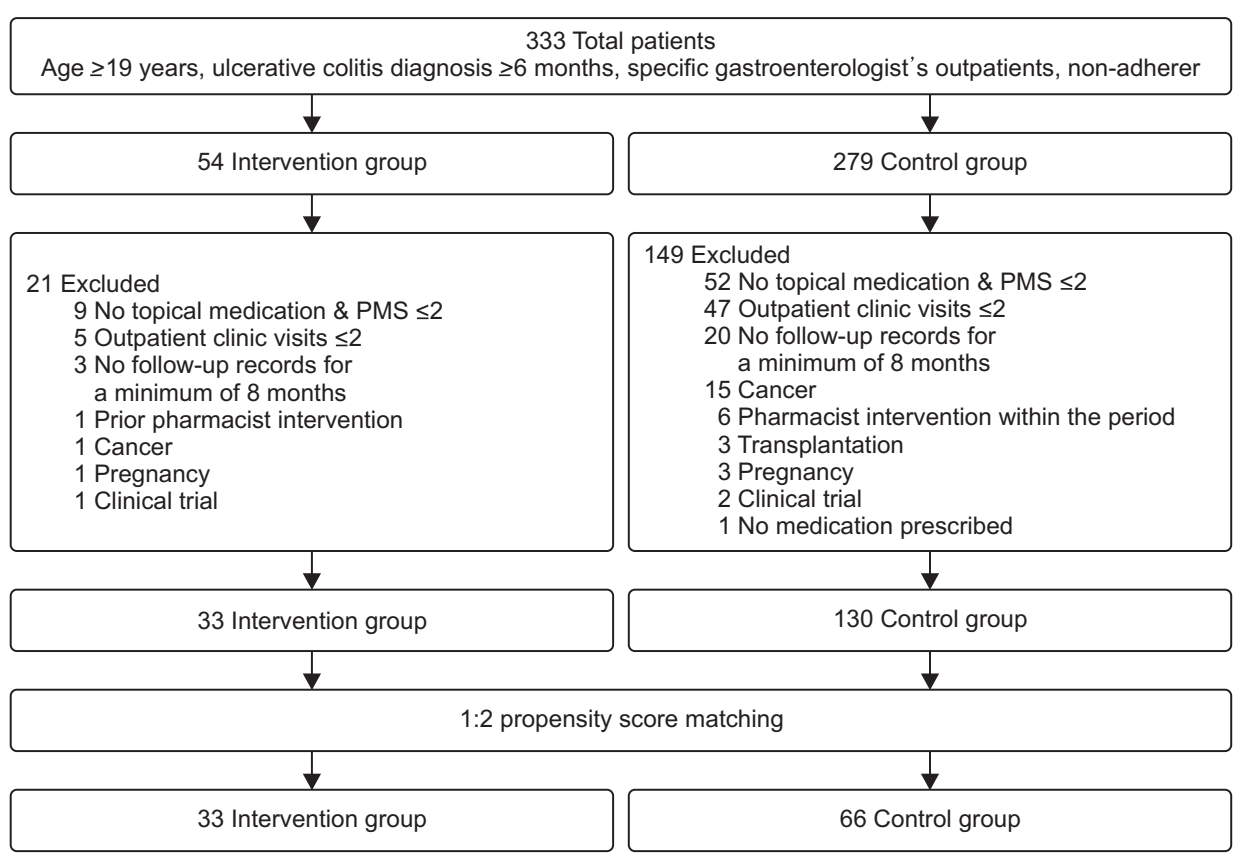

Fig. 2. Flow diagram depicting the selection of the study population. PMS, partial Mayo score. 
Table 1. Demographics and Baseline Characteristics of the Study Population

\begin{tabular}{|c|c|c|c|c|}
\hline Classification & Control $(n=66)$ & Intervention ( $\mathrm{n}=33$ ) & Total (n=99) & $\mathrm{p}$-value \\
\hline Male sex* & $40(60.6)$ & $20(60.6)$ & $60(60.6)$ & 1.000 \\
\hline Age, yr* & $32.6(26.3-40.6)$ & $33.0(25.1-42.6)$ & $33.0(26.1-41.5)$ & 0.827 \\
\hline Age at diagnosis, yr* & $29.0(22.5-35.9)$ & $29.1(23.7-40.2)$ & $29.1(22.7-36.6)$ & 0.670 \\
\hline Age at diagnosis group & & & & 0.472 \\
\hline$\leq 16 \mathrm{yr}$ & 0 & 0 & 0 & \\
\hline 17 to $<40 \mathrm{yr}$ & 54 (81.8) & 25 (75.8) & 79 (79.8) & \\
\hline$\geq 40 \mathrm{yr}$ & 12 (18.2) & 8 (24.2) & $20(20.2)$ & \\
\hline Duration of diagnosis, yr* & $3.5(1.3-4.9)$ & $3.7(1.8-5.0)$ & $3.6(1.5-4.9)$ & 0.879 \\
\hline Smoking status & & & & 0.264 \\
\hline Never & 46 (69.7) & 25 (75.8) & $71(71.7)$ & \\
\hline Former & 20 (30.3) & 7 (21.2) & 27 (27.3) & \\
\hline Current & 0 & $1(3.0)$ & $1(1.0)$ & \\
\hline Disease extent ${ }^{*}{ }^{\dagger}$ & & & & 0.693 \\
\hline E1 & 18 (27.3) & $7(21.2)$ & 25 (25.3) & \\
\hline E2 & 29 (43.9) & $14(42.4)$ & $43(43.4)$ & \\
\hline E3 & $19(28.8)$ & $12(36.4)$ & 31 (31.3) & \\
\hline PMS* & $1.5(0.0-3.0)$ & $2.0(0.0-3.0)$ & $2.0(0.0-3.0)$ & 0.194 \\
\hline $\mathrm{PMS} \leq 2^{*}$ & $47(71.2)$ & $21(63.6)$ & $68(68.7)$ & 0.444 \\
\hline 1 yr prior hospitalization: yes & $4(6.1)$ & $2(6.1)$ & $6(6.1)$ & 1.000 \\
\hline Prior ER visit: yes & $5(7.6)$ & $5(15.2)$ & $10(10.1)$ & 0.294 \\
\hline 1 yr prior corticosteroid use: yes & 14 (21.2) & $4(12.1)$ & $18(18.2)$ & 0.269 \\
\hline Prior surgery: yes & 0 & $1(3.0)$ & $1(1.0)$ & 0.333 \\
\hline Prior appendectomy: yes & $2(3.0)$ & $1(3.0)$ & $3(3.0)$ & 1.000 \\
\hline Anemia: yes & $11(16.7)$ & $5(15.2)$ & $16(16.2)$ & 0.847 \\
\hline Insomnia: yes & $1(1.5)$ & $3(9.1)$ & $4(4.0)$ & 0.107 \\
\hline Depression: yes & $1(1.5)$ & 0 & $1(1.0)$ & 1.000 \\
\hline Vitamin D deficiency: no & $66(100.0)$ & $33(100.0)$ & $99(100.0)$ & 1.000 \\
\hline Diabetes mellitus: yes & $1(1.5)$ & $1(3.0)$ & $2(2.0)$ & 1.000 \\
\hline Dyslipidemia: yes & $3(4.5)$ & $2(6.1)$ & $5(5.1)$ & 1.000 \\
\hline \multicolumn{5}{|l|}{ Current medications } \\
\hline 5-ASA: yes & $66(100.0)$ & $33(100.0)$ & $99(100.0)$ & 1.000 \\
\hline Oral use* & $66(100.0)$ & $33(100.0)$ & $99(100.0)$ & 1.000 \\
\hline Topical use & $63(95.5)$ & 31 (93.9) & $94(94.9)$ & 1.000 \\
\hline 6-MP: yes & $3(4.5)$ & 0 & $3(3.0)$ & 0.549 \\
\hline Azathioprine: yes & 14 (21.2) & $6(18.2)$ & $20(20.2)$ & 0.723 \\
\hline MTX: no & $66(100.0)$ & $33(100.0)$ & $99(100.0)$ & 1.000 \\
\hline Corticosteroids: yes & $6(9.1)$ & $1(3.0)$ & $7(7.1)$ & 0.419 \\
\hline Biologics: yes* & $6(9.1)$ & $6(18.2)$ & $12(12.1)$ & 0.207 \\
\hline Infliximab & 0 & $4(12.1)$ & $4(4.0)$ & \\
\hline Adalimumab & $3(4.5)$ & $1(3.0)$ & $4(4.0)$ & \\
\hline Golimumab & $2(3.0)$ & $1(3.0)$ & $3(3.0)$ & \\
\hline Vedolizumab & $1(1.5)$ & 0 & $1(1.0)$ & \\
\hline
\end{tabular}

Data are presented as the number (\%) or median (interquartile range).

PMS, partial Mayo score; ER, emergency room; 5-ASA, 5-aminosalicylate; 6-MP, 6-mercaptopurine; MTX, methotrexate.

*Matched variables in the intervention group; ${ }^{\dagger}$ Disease extent of ulcerative colitis (UC) was defined as ulcerative proctitis (E1), left-sided UC (E2), or extensive UC (E3).

However, the MPR for the observation period of 6 months before and after the baseline increased significantly in the intervention group alone $(\mathrm{n}=65$, from $73.4 \%$ to $87.9 \%, \mathrm{p}=0.070$ in the control group vs $\mathrm{n}=33$, from $60.5 \%$ to $83.6 \%, \mathrm{p}=0.004$ in the intervention group) (Fig. 3). Additionally, the rate of nonadherence significantly decreased from $60.6 \%$ to $30.3 \%$ in the intervention group alone $(\mathrm{p}=0.013)$. In the control group, the nonadherence rate decreased from $36.9 \%$ to $21.5 \%$ ( $\mathrm{p}=0.054$ ) (Fig. 4).
There was a significant difference between the two groups regarding the MPRs $(73.4 \%$ vs $60.5 \% ; \mathrm{p}=0.023)$ and nonadherence rates $(36.9 \%$ vs $60.6 \% ; \mathrm{p}=0.026$ ) for the 6 -month observation period before the baseline. One of the $66 \mathrm{pa}-$ tients in the control group was excluded owing to a lack of prescribing information in the 6 months before baseline. Although changes in the MPR (delta rates) between the two groups did not show a significant difference between 6 months before and after the baseline $(9.5 \%$ in the control 
Table 2. Clinical Outcomes

\begin{tabular}{|c|c|c|c|c|}
\hline Classification & Control $(n=66)$ & Intervention (n=33) & Total (n=99) & $\mathrm{p}$-value \\
\hline Flare-up & $36(54.6)$ & 11 (33.3) & $47(47.5)$ & 0.046 \\
\hline Dose escalation & $27(40.9)$ & 9 (27.3) & $36(36.4)$ & 0.184 \\
\hline Step-up therapy & $8(12.1)$ & $4(12.1)$ & $12(12.1)$ & 1.000 \\
\hline Severe activity (PMS $\geq 5$ ) & $14(21.2)$ & 5 (15.2) & 19 (19.2) & 0.470 \\
\hline Corticosteroid use & $17(25.8)$ & $4(12.1)$ & $21(21.2)$ & 0.118 \\
\hline MPR, \% (observation of $1 \mathrm{yr}$ ) & $90.8(77.1-100.0)$ & $86.3(75.2-96.2)$ & $89.6(76.7-99.7)$ & 0.254 \\
\hline Nonadherence (observation of $1 \mathrm{yr}$ ) & $20(30.3)$ & $13(39.4)$ & 33 (33.3) & 0.366 \\
\hline DDD of corticosteroids & $20.4 \pm 41.7$ & $12.6 \pm 38.3$ & $17.8 \pm 40.5$ & 0.158 \\
\hline Hospitalization & $1(1.5)$ & 0 & $1(1.0)$ & 1.000 \\
\hline Emergency room visit & 0 & $1(3.0)$ & $1(1.0)$ & 0.333 \\
\hline
\end{tabular}

Data are presented as the number (\%), median (interquartile range), or mean \pm SD.

PMS, partial Mayo score; MPR, medication possession ratio; DDD, defined daily dose.

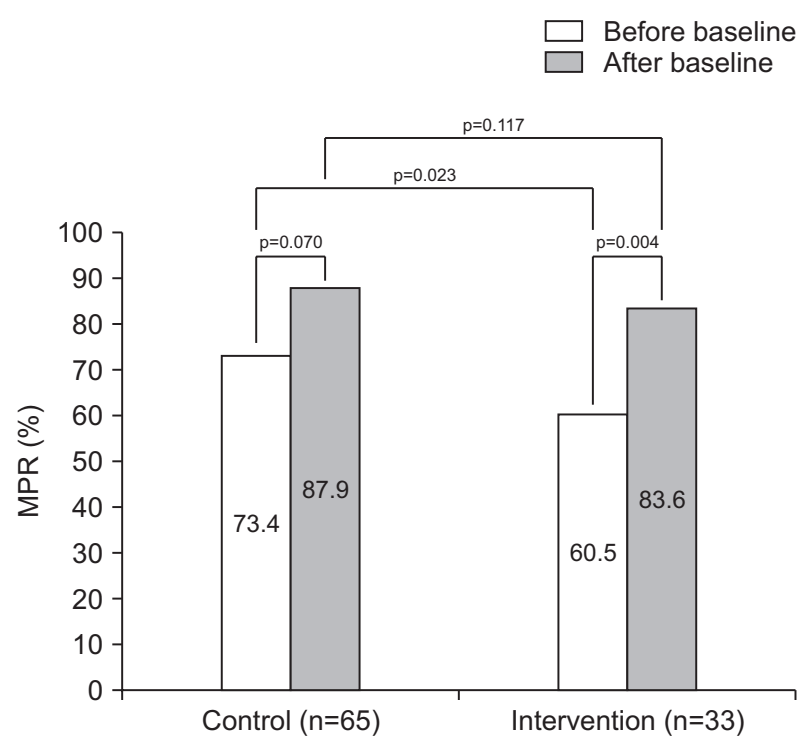

Fig. 3. Medication possession ratio (MPR) before and after the baseline for an observation period of 6 months. The MPR for the observation period of 6 months before and after the baseline increased significantly in the intervention group $(n=65$, from $73.4 \%$ to $87.9 \%$; $p=0.070$ in the control group vs $n=33$, from $60.5 \%$ to $83.6 \% ; p=0.004$ in the intervention group). One of 66 patients was excluded from the control group due to the lack of exact prescription history because of transfer from another hospital.

group vs $23.1 \%$ in the intervention group; $\mathrm{p}=0.150$ ), the MPR changed between 6 months before and 1 year after the baseline were significantly increased in the intervention group compared to those in the control group ( $7.1 \%$ in the control group vs $23.3 \%$ in the intervention group; $\mathrm{p}=0.013$ ).

\section{Comparison of disease activity (PMS) after pharmacist intervention}

A comparison of the group-by-time interaction regarding the PMS at 1-month intervals revealed a statistically significant difference between the two groups ( $\mathrm{p}=0.002$ )

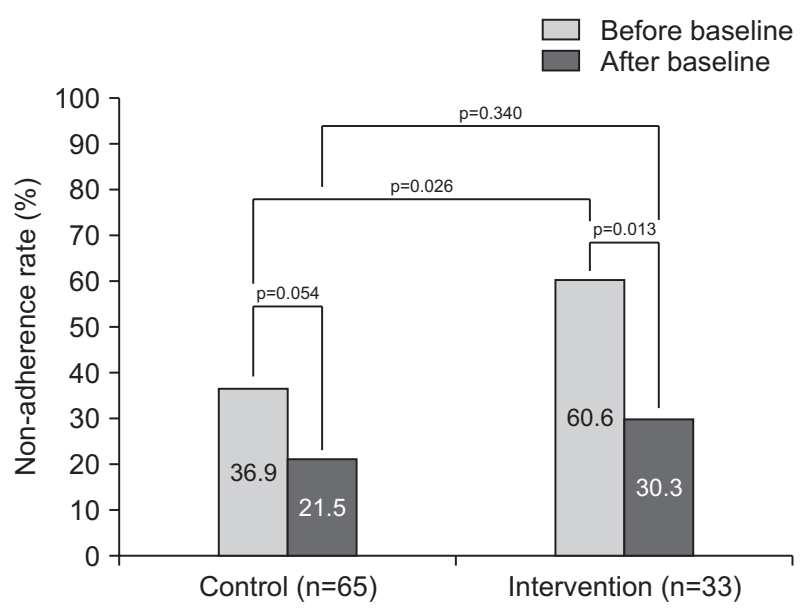

Fig. 4. The rates of nonadherence before and after the baseline for an observation period of 6 months. The rate of nonadherence significantly decreased from $60.6 \%$ to $30.3 \%$ in the intervention group $(p=0.013$ ). In the control group, the rate of nonadherence decreased from $36.9 \%$ to $21.5 \%$ ( $p=0.054)$. One of 66 patients was excluded from the control group due to the lack of exact prescription history because of transfer from another hospital.

(Fig. 5). Linear regression analysis revealed that the control group did not exhibit any significant correlation between time and the PMS $\left(\mathrm{r}^{2}<0.001, \mathrm{p}=0.669\right)$. However, the PMS decreased by 0.094 every month in the intervention group, revealing a significant correlation between time and the PMS $\left(\mathrm{r}^{2}=0.035, \mathrm{p}=0.013\right)$.

\section{Comparison of the disease flare-up incidence}

There was a significant difference between the two groups concerning the incidence of flare-ups during the follow-up period (control group, $54.6 \%$ vs intervention group, $33.3 \%$; $\mathrm{p}=0.046$ ). However, there were no significant differences amongst the sub-items concerning the occurrence of flare-up, such as dose escalation $(\mathrm{p}=0.184)$, stepup therapy $(\mathrm{p}=1.000)$, severe disease activity $(\mathrm{p}=0.470)$, or additional corticosteroid usage $(\mathrm{p}=0.118)$ (Table 2$)$. The 
survival rates of the patients free from any flare-up incidents were $38.8 \%$ and $66.7 \%$ in the control and intervention groups, respectively. This difference was not statistically significant $\left(\chi^{2}=1.685, p=0.194\right)$ (Supplementary Fig. $1)$.

\section{Comparisons of other items}

The mean of corticosteroid consumption in defined daily dose for 1 year was $20.4 \pm 41.7$ and $12.6 \pm 38.3$ in the control and intervention groups, respectively $(\mathrm{p}=0.158)$. There was no significant difference between the control and intervention groups regarding the rates of hospitalization during the follow-up period (control group, $1.5 \%$ vs intervention group, $0 \% ; \mathrm{p}=1.000$ ). None of the patients in either group underwent surgery. Finally, only one patient in the intervention group visited the emergency room (Table 2).

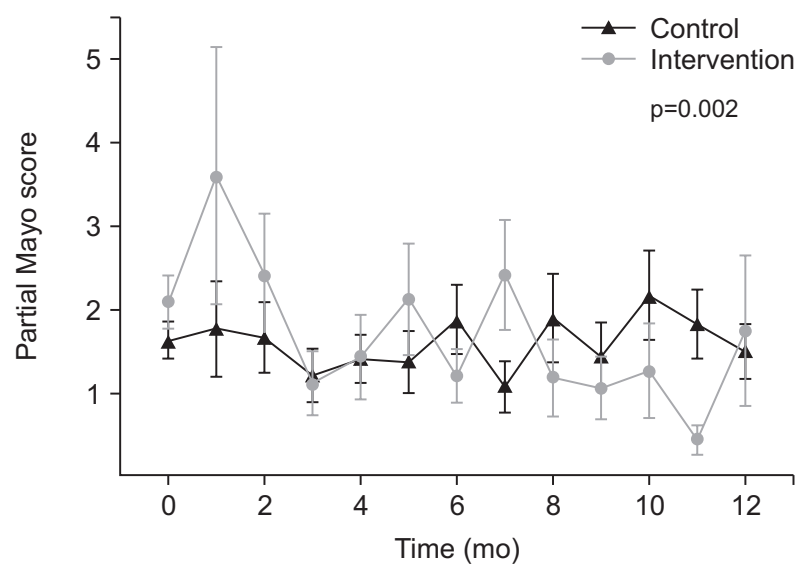

Fig. 5. Comparison of the group-by-time interaction in the partial Mayo score (PMS) during the course of the 1-year follow-up using the linear mixed model analysis. This analysis revealed a statistically significant difference between the two groups $(p=0.002)$. The values are expressed as the mean \pm standard errors of the PMS monthly.

\section{Factors related to flare-up}

Multivariate logistic regression revealed that pharmacist intervention (adjusted odds ratio [OR], 0.370; 95\% confidence interval, 0.145 to $0.945 ; \mathrm{p}=0.038$ ), the baseline PMS (adjusted OR, 1.333; 95\% confidence interval, 1.039 to 1.710; $\mathrm{p}=0.024$ ), and history of 1-year prior corticosteroid use (adjusted OR, 3.312; 95\% confidence interval, 1.035 to 10.597; $\mathrm{p}=0.044$ ) were independent factors associated with flare up-free survival (Table 3 ).

\section{DISCUSSION}

This was the first study to evaluate the impact on clinical outcomes and medication adherence of pharmacist intervention in patients with UC and low medication adherence. Although pharmacist intervention for patients with IBD has been shown to improve medication adherence, few studies have evaluated its effects on clinical outcomes. ${ }^{19,20}$ The results of this study revealed that nonadherence rate, disease activity over time, and flare-ups were significantly reduced in the intervention group alone. This study confirmed that pharmacist intervention could be one solution for improving patient outcomes by increasing medication adherence through individually educating patients with IBD. The pharmacist's role is emphasized in the field of IBD treatment in international guidelines and agreements. ${ }^{21,27}$ Nonetheless, the participation of pharmacists as members of the IBD multidisciplinary team is not yet common in South Korea. Hence, this study supports the guidelines and agrees with the necessity for the active involvement of expert pharmacists in the management of IBD patients.

Several systematic literature reviews and meta-analysis studies have demonstrated that pharmacist interventions for patients with diabetes, depression, and autoimmune diseases improved their medication adherence. ${ }^{16-18}$

Table 3. Logistic Regression Model to Identify the Factors Influencing "Flare-Up" in Univariate and Multivariate Analyses (n=99)

\begin{tabular}{|c|c|c|c|c|c|}
\hline \multirow{2}{*}{ Factor } & \multicolumn{2}{|c|}{ Univariate analysis } & \multicolumn{3}{|c|}{ Multivariate analysis } \\
\hline & $\mathrm{OR}(95 \% \mathrm{Cl})$ & p-value & Estimate & OR $(95 \% \mathrm{Cl})$ & $\mathrm{p}$-value \\
\hline Intervention (intervention group vs control group) & $0.417(0.174-0.996)$ & 0.049 & -0.995 & $0.370(0.145-0.945)$ & 0.038 \\
\hline Sex (male vs female) & $0.464(0.204-1.054)$ & 0.067 & - & - & - \\
\hline Baseline PMS & $1.277(1.009-1.616)$ & 0.042 & 0.287 & $1.333(1.039-1.710)$ & 0.024 \\
\hline Baseline remission (yes vs no) & $0.442(0.185-1.054)$ & 0.066 & - & - & - \\
\hline Topical 5-ASA use at the baseline (yes vs no) & $0.211(0.023-1.957)$ & 0.171 & - & - & - \\
\hline AZA use at baseline (yes vs nol & 3.253 (1.132-9.348) & 0.029 & - & - & - \\
\hline Biologics use at baseline (yes vs no) & $2.462(0.690-8.786)$ & 0.165 & - & - & - \\
\hline Corticosteroids use at baseline (yes vs no) & $7.463(0.864-64.494)$ & 0.068 & - & - & - \\
\hline Corticosteroid use before 1 year (yes vs no) & $3.594(1.171-11.036)$ & 0.025 & 1.197 & $3.312(1.035-10.597)$ & 0.044 \\
\hline
\end{tabular}

Method=forward stepwise regression.

OR, odds ratio; $\mathrm{Cl}$, confidence interval; PMS, partial Mayo score; 5-ASA, 5-aminosalicylate; AZA, azathioprine. 
Moreover, a previous study verified that participants who received face-to-face intervention from the pharmacist exhibited improved quality of life, knowledge regarding their medications, physical function, and symptoms compared with the participants who did not. ${ }^{28}$

Furthermore, pharmacist intervention as part of an outpatient IBD multidisciplinary team approach showed high medication adherence by participants. ${ }^{20}$ Previous studies assessing pharmacist intervention in various disease groups, including IBD, concur with our results, which revealed a significant improvement in medication adherence in the intervention group alone. Moreover, in situations of remission, patients are often uncertain about the need for therapeutic agents, with their medication adherence displaying a consequent decline. ${ }^{29}$ Therefore, using the PSM, both groups were comprised so that there was no difference regarding disease status when patients were enrolled in this study. The intervention group alone displayed improved medication adherence, thereby confirming that pharmacist intervention was an important factor in remission maintenance. Nevertheless, since the data were calculated based on an observation period of only 6 months and there was a significant difference in the MPR and nonadherence rates 6 months before the baseline between the two groups, it is necessary to confirm these results in larger follow-up studies.

This study used PMS to evaluate the effect of pharmacist intervention on disease activity, which emphasizes its relevance. This study also identified group-by-time interactions in their PMS using linear mixed model statistical analysis at monthly intervals and found a statistically significant difference between the two groups ( $\mathrm{p}=0.002$ ). A correlation was observed in the intervention group alone, with a monthly decrease in the PMS by $0.094(\mathrm{p}=0.013)$. This confirmed that pharmacist intervention contributed to the reduction in disease activity.

Ardizzone et al. ${ }^{30}$ reported that corticosteroid therapy was a risk factor for the frequent development of flare-ups. Patients with UC with a history of corticosteroid usage had a higher risk of clinical flare-ups. ${ }^{31}$ Furthermore, $65 \%$ of the patients developed flare-ups that warranted corticosteroid therapy within 2 years after the initial corticosteroid usage. ${ }^{32}$ We observed an increase in the risk of flare-ups with 1-year prior corticosteroid use, which concurred with the results of previous studies.

This study assessed an increase in the PMS at the baseline as a predictive factor for the increase in the incidence of flare-ups. Indeed, two-thirds of patients who were in a non-remission state at the baseline developed flare-ups over 1 year. These findings were similar to that of a previous study reporting an association between an increase in the PMS and an increased risk of adverse physicianreported clinical outcomes, including current flare-ups. ${ }^{33}$

Although there is no consensus regarding the definition of a clinical flare-up in UC, the basis of the clinical diagnosis of a flare-up is the manifestation of symptoms. ${ }^{34}$ Therefore, the definition of the occurrence of a flare-up in this study included additional corticosteroid usage, the occurrence of dose escalation, step-up therapy for UC compared with previous prescriptions, or severe disease activity.

This study had some limitations. First, although the PSM method selected a control group similar to the intervention group, significant differences were found regarding MPR and nonadherence rate for 6 months before the baseline between the two groups due to limitations inherent to the retrospective study method. To overcome this limitation, we compared and analyzed the changes in the MPR (delta rates) between 6 months before and 1 year after baseline. Although the control group showed no significant difference, the intervention group showed a significant increase in the delta rate. Second, the number of non-adherers identified in the EMRs at the baseline was only half of those revealed via the MPR. The adherence of patients undergoing treatment may include not only the administration of prescribed medications but also regular appointments and scheduled laboratory test completion. ${ }^{15}$ It is presumed that patients defined by the doctor as being nonadherent in the EMRs included those who were non-compliant in other aspects, in addition to being low medication adherence. However, it is estimated that this difference was not significant, as the MPR reflects the impact of scheduled appointment adherence failure. Moreover, as the medication adherence was calculated using the MPR, it was not possible to confirm whether the patient took their medications. Third, this study was retrospective; hence, the medication adherence was confirmed using the MPR alone. Fourth, the population size was relatively small. Fifth, due to the limitations of this retrospective study, regular endoscopy records could not be obtained. Thus, confirmation of healing of mucosal lesions was not possible. Sixth, since the study involved a review of EMRs, the analysis of the factors that can affect flare-ups did not include marital status, economic level, education level, or mental status. Finally, a bias might have occurred regarding the control group due to other factors that were not applied to the covariate. As a consequence of the basic statistical characteristics, there was no statistical difference regarding the other factors. A logistic regression analysis was performed, applying other factors to the covariate to minimize any potential bias. Thus, potential differences between the two groups can be considered to have been minimized, though not eliminated. Future multicenter, 
prospective studies should be performed to overcome the abovementioned limitations and to generalize the results of this study.

In conclusion, to our knowledge, this was the first study to analyze the effect of pharmacist intervention on medication adherence and clinical outcomes in patients with UC and low medication adherence. This study revealed that pharmacist intervention had a positive effect on remission maintenance, as the intervention group displayed significant improvement in medication adherence, improvement in disease activity, and reduction in flare-up incidence. Further, pharmacist intervention reduced the risk of flareup incidences. Therefore, this study suggested that patients with UC and low adherence may require pharmacist intervention to improve their medication adherence and clinical outcomes.

\section{CONFLICTS OF INTEREST}

J.H.C. is an editorial board member of the journal but was not involved in the peer reviewer selection, evaluation, or decision process of this article. No other potential conflicts of interest relevant to this article were reported.

\section{AUTHOR CONTRIBUTIONS}

Study concept and design: J.S.K., J.H.C., K.H.K. Data acquisition: J.S.K. Data analysis and interpretation: J.S.K., J.H.C., K.H.K. Drafting of the manuscript: J.S.K. Critical revision of the manuscript for important intellectual content: J.S.K., M.J.G., E.S.S., Y.M.Y., J.H.C., K.H.K. Data analysis: J.S.K., M.J.G. Statistical analysis: J.S.K., Y.M.Y. Administrative, technical, or material support: J.S.K., M.J.G., E.S.S. Study supervision: J.H.C., K.H.K. Approval of final manuscript: all authors.

\section{ORCID}

Jae Song Kim

Min Jung Geum

Eun Sun Son

Yun Mi Yu

Jae Hee Cheon

Kyeng Hee Kwon https://orcid.org/0000-0002-3532-3892

https://orcid.org/0000-0001-8493-4078

https://orcid.org/0000-0002-3905-532X

https://orcid.org/0000-0002-8267-9453

https://orcid.org/0000-0002-2282-8904

https://orcid.org/0000-0003-2421-4030

\section{SUPPLEMENTARY MATERIALS}

Supplementary materials can be accessed at https://doi. org/10.5009/gnl210371.

\section{REFERENCES}

1. Feuerstein JD, Cheifetz AS. Ulcerative colitis: epidemiology, diagnosis, and management. Mayo Clin Proc 2014;89:15531563.

2. Mizoguchi E, Low D, Ezaki Y, Okada T. Recent updates on the basic mechanisms and pathogenesis of inflammatory bowel diseases in experimental animal models. Intest Res 2020;18:151-167.

3. Park J, Cheon JH. Incidence and prevalence of inflammatory bowel disease across Asia. Yonsei Med J 2021;62:99-108.

4. Lee J, Im JP, Han K, et al. Changes in direct healthcare costs before and after the diagnosis of inflammatory bowel disease: a nationwide population-based study. Gut Liver 2020;14:89-99.

5. Lee SH, Chang K, Seo KS, et al. Changes in prevalence and perception of complementary and alternative medicine use in Korean inflammatory bowel disease patients: results of an 8-year follow-up survey. Intest Res 2020;18:192-199.

6. Gibson PR, Vaizey C, Black CM, et al. Relationship between disease severity and quality of life and assessment of health care utilization and cost for ulcerative colitis in Australia: a cross-sectional, observational study. J Crohns Colitis 2014;8:598-606.

7. Oh SJ, Shin GY, Soh H, et al. Long-term outcomes of infliximab in a real-world multicenter cohort of patients with acute severe ulcerative colitis. Intest Res 2021;19:323-331.

8. Rubin DT, Ananthakrishnan AN, Siegel CA, Sauer BG, Long MD. ACG clinical guideline: ulcerative colitis in adults. Am J Gastroenterol 2019;114:384-413.

9. Hibi T, Kamae I, Pinton P, et al. Efficacy of biologic therapies for biologic-naïve Japanese patients with moderately to severely active ulcerative colitis: a network meta-analysis. Intest Res 2021;19:53-61.

10. Solberg IC, Lygren I, Jahnsen J, et al. Clinical course during the first 10 years of ulcerative colitis: results from a population-based inception cohort (IBSEN Study). Scand J Gastroenterol 2009;44:431-440.

11. Chan W, Chen A, Tiao D, Selinger C, Leong R. Medication adherence in inflammatory bowel disease. Intest Res 2017;15:434-445.

12. Testa A, Castiglione F, Nardone OM, Colombo GL. Adherence in ulcerative colitis: an overview. Patient Prefer Adherence 2017;11:297-303.

13. Jackson CA, Clatworthy J, Robinson A, Horne R. Factors as- 
sociated with non-adherence to oral medication for inflammatory bowel disease: a systematic review. Am J Gastroenterol 2010;105:525-539.

14. Burisch J, Jess T, Martinato M, Lakatos PL; ECCO-EpiCom. The burden of inflammatory bowel disease in Europe. J Crohns Colitis 2013;7:322-337.

15. Herman ML, Kane SV. Treatment nonadherence in inflammatory bowel disease: identification, scope, and management strategies. Inflamm Bowel Dis 2015;21:2979-2984.

16. Readdean KC, Heuer AJ, Scott Parrott J. Effect of pharmacist intervention on improving antidepressant medication adherence and depression symptomology: a systematic review and meta-analysis. Res Social Adm Pharm 2018;14:321-331.

17. Presley B, Groot W, Pavlova M. Pharmacy-led interventions to improve medication adherence among adults with diabetes: a systematic review and meta-analysis. Res Social Adm Pharm 2019;15:1057-1067.

18. Sah SK, R S, Ramesh M, Chand S. Impact of pharmacist care in the management of autoimmune disorders: a systematic review of randomized control trials and non-randomized studies. Res Social Adm Pharm 2021;17:1532-1545.

19. Tiao DK, Chan W, Jeganathan J, et al. Inflammatory bowel disease pharmacist adherence counseling improves medication adherence in Crohn's disease and ulcerative colitis. Inflamm Bowel Dis 2017;23:1257-1261.

20. Khiani V, Stubbings J. The pharmacist's role in biologic management for IBD in a health system-integrated practice model. Am J Pharm Benefits 2015;7:215-220.

21. Fiorino G, Lytras T, Younge L, et al. Quality of care standards in inflammatory bowel diseases: a European Crohn's and Colitis Organisation [ECCO] position paper. J Crohns Colitis $2020 ; 14: 1037-1048$.

22. Parikh A, Fox I, Leach T, et al. Long-term clinical experience with vedolizumab in patients with inflammatory bowel disease. Inflamm Bowel Dis 2013;19:1691-1699.

23. Lewis JD, Chuai S, Nessel L, Lichtenstein GR, Aberra FN, Ellenberg JH. Use of the noninvasive components of the Mayo score to assess clinical response in ulcerative colitis. Inflamm Bowel Dis 2008;14:1660-1666.

24. Kuriyama M, Kato J, Takemoto K, Hiraoka S, Okada H, Yamamoto K. Prediction of flare-ups of ulcerative colitis using quantitative immunochemical fecal occult blood test. World J Gastroenterol 2010;16:1110-1114.

25. Baumgart DC, Bokemeyer B, Drabik A, Stallmach A, Schreiber S; Vedolizumab Germany Consortium. Vedolizumab induction therapy for inflammatory bowel disease in clinical practice: a nationwide consecutive German cohort study. Aliment Pharmacol Ther 2016;43:1090-1102.

26. WHO Collaborating Centre for Drug Statistics Methodology. ATC Classification Index with DDDs [Internet]. Oslo: WHO Collaborating Centre for Drug Statistics Methodology; c2020 [cited 2021 Mar 6]. Available from: www.whocc. no/atc_ddd_index/.

27. Kapasi R, Glatter J, Lamb CA, et al. Consensus standards of healthcare for adults and children with inflammatory bowel disease in the UK. Frontline Gastroenterol 2019;11:178-187.

28. Conn VS, Ruppar TM, Enriquez M, Cooper PS. Patient-centered outcomes of medication adherence interventions: systematic review and meta-analysis. Value Health 2016;19:277285.

29. LaRosa JC. Poor compliance: the hidden risk factor. Curr Atheroscler Rep 2000;2:1-4.

30. Ardizzone S, Petrillo M, Molteni P, Desideri S, Bianchi Porro G. Coated oral 5-aminosalicylic acid (Claversal) is equivalent to sulfasalazine for remission maintenance in ulcerative colitis: a double-blind study. J Clin Gastroenterol 1995;21:287289.

31. Fukuda T, Naganuma M, Sugimoto S, et al. The risk factor of clinical relapse in ulcerative colitis patients with low dose 5-aminosalicylic acid as maintenance therapy: a report from the IBD registry. PLoS One 2017;12:e0187737.

32. Khan N, Abbas A, Williamson A, Balart L. Prevalence of corticosteroids use and disease course after initial steroid exposure in ulcerative colitis. Dig Dis Sci 2013;58:2963-2969.

33. Naegeli AN, Hunter T, Dong Y, et al. Full, partial, and modified permutations of the Mayo score: characterizing clinical and patient-reported outcomes in ulcerative colitis patients. Crohns Colitis 360 2021;3:otab007.

34. Li J, Zhao X, Li X, Lu M, Zhang H. Systematic review with meta-analysis: fecal calprotectin as a surrogate marker for predicting relapse in adults with ulcerative colitis. Mediators Inflamm 2019;2019:2136501. 